\title{
Quand diversité ethnoculturelle et défavorisation se conjuguent au quotidien : regards croisés d'acteurs en contexte scolaire montréalais sur les défis et les leviers d'intervention
}

\section{Justine Gosselin-Gagné}

Volume 8, numéro 2, 2018

L'école à l'épreuve de la diversité ethnoculturelle de son personnel : regards compréhensifs croisés

URI : https://id.erudit.org/iderudit/1066955ar

DOI : https://doi.org/10.7202/1066955ar

Aller au sommaire du numéro

Éditeur(s)

Alterstice

ISSN

1923-919X (numérique)

Découvrir la revue

Citer cet article

Gosselin-Gagné, J. (2018). Quand diversité ethnoculturelle et défavorisation se conjuguent au quotidien : regards croisés d'acteurs en contexte scolaire montréalais sur les défis et les leviers d'intervention. Alterstice, 8(2), 89-100. https://doi.org/10.7202/1066955ar

\section{Résumé de l'article}

La diversité sociale et ethnoculturelle est de plus en plus présente dans certains milieux scolaires du Québec, une réalité qui soulève différents enjeux. L'école est appelée à s'adapter à cette diversité, en mettant à profit au quotidien les savoirs, les savoir-être et les savoir-faire de différents acteurs. Plusieurs perspectives théoriques ont été proposées dans ce contexte : éducation multiculturelle, éducation interculturelle, antiracisme, éducation à la citoyenneté, etc. Ma recherche s'inscrit dans un courant théorique auquel de plus en plus de chercheurs s'intéressent : le paradigme de l'éducation inclusive. Pendant une année scolaire, je me suis intégrée au quotidien de deux écoles primaires montréalaises en contexte de défavorisation socioéconomique et de pluriethnicité. L'approche ethnographique adoptée m'a donné l'opportunité d'accéder aux " coulisses » de ces milieux qui se mobilisent, au quotidien, pour s'adapter aux besoins de leur public scolaire. Les diverses méthodes employées (observations, entretiens individuels et de groupe, etc.) m'ont permis de construire un corpus de données qui favorise le croisement du regard de différents acteurs. Les résultats se structurent autour de quatre thèmes principaux : leadership de la direction, climat socioprofessionnel et pratiques de l'équipe-école, relations écoles-familles et collaborations avec les ressources communautaires. Ces thèmes renvoient à diverses portes d'entrée permettant aux écoles participantes de se mobiliser pour répondre aux besoins d'élèves qui cumulent diverses vulnérabilités socioscolaires, afin de soutenir leur réussite éducative et, ainsi, de mettre en oeuvre une éducation inclusive. 


\title{
7
}

\section{Quand diversité ethnoculturelle et défavorisation se conjuguent au quotidien : regards croisés d'acteurs en contexte scolaire montréalais sur les défis et les leviers d'intervention}

Justine Gosselin-Gagné ${ }^{1}$

\begin{abstract}
Résumé
La diversité sociale et ethnoculturelle est de plus en plus présente dans certains milieux scolaires du Québec, une réalité qui soulève différents enjeux. L'école est appelée à s'adapter à cette diversité, en mettant à profit au quotidien les savoirs, les savoir-être et les savoir-faire de différents acteurs. Plusieurs perspectives théoriques ont été proposées dans ce contexte : éducation multiculturelle, éducation interculturelle, antiracisme, éducation à la citoyenneté, etc. Ma recherche s'inscrit dans un courant théorique auquel de plus en plus de chercheurs s'intéressent : le paradigme de l'éducation inclusive. Pendant une année scolaire, je me suis intégrée au quotidien de deux écoles primaires montréalaises en contexte de défavorisation socioéconomique et de pluriethnicité. L'approche ethnographique adoptée m'a donné l'opportunité d'accéder aux "coulisses" de ces milieux qui se mobilisent, au quotidien, pour s'adapter aux besoins de leur public scolaire. Les diverses méthodes employées (observations, entretiens individuels et de groupe, etc.) m'ont permis de construire un corpus de données qui favorise le croisement du regard de différents acteurs. Les résultats se structurent autour de quatre thèmes principaux : leadership de la direction, climat socioprofessionnel et pratiques de l'équipe-école, relations écolesfamilles et collaborations avec les ressources communautaires. Ces thèmes renvoient à diverses portes d'entrée permettant aux écoles participantes de se mobiliser pour répondre aux besoins d'élèves qui cumulent diverses vulnérabilités socioscolaires, afin de soutenir leur réussite éducative et, ainsi, de mettre en œuvre une éducation inclusive.
\end{abstract}

\section{Rattachement de l'auteure}

${ }^{1}$ Université du Québec à Montréal, Montréal, Canada

\section{Correspondance}

gosselin-gagne.justine@courrier.uqam.ca

\section{Mots clés}

diversité ethnoculturelle, immigration récente, défavorisation socioéconomique, éducation inclusive, ethnographie scolaire

\section{Pour citer cet article}

Gosselin-Gagné, J. (2018). Quand diversité ethnoculturelle et défavorisation se conjuguent au quotidien : regards croisés d'acteurs en contexte scolaire montréalais sur les défis et les leviers d'intervention. Alterstice, 8(2), 89-100. 


\section{La diversité dans les écoles montréalaises}

Certaines écoles de Montréal sont caractérisées aujourd’hui par une grande diversité ethnoculturelle (incluant ici diversité linguistique et diversité religieuse), nourrie au fil du temps par l'enchaînement de différents flux migratoires. Les défis que génère cette réalité appellent les différents praticiens scolaires à se mobiliser afin de mettre en œuvre des interventions et de poursuivre un objectif de soutien à la réussite de tous. Cet article fait état des principaux résultats de ma recherche doctorale, dont l'objectif était de brosser le portrait d'écoles primaires montréalaises en contexte de défavorisation et de pluriethnicité qui se mobilisent pour soutenir la réussite des élèves.

Dans les lignes qui suivent est d'abord exposée la problématique au cœur de cette recherche, puis un bref survol d'écrits concernant l'éducation inclusive est effectué. Ensuite, le cadre méthodologique est présenté et les résultats exposés. Ces derniers illustrent différents défis auxquels des écoles en milieu défavorisé et pluriethnique font face au quotidien. Ils mettent également en lumière divers leviers pouvant être investis afin de contrecarrer les défis nommés précédemment. En conclusion, un résumé de la recherche est effectué et quelques limites sont mentionnées, tout en proposant plusieurs perspectives de recherche.

\section{Soutenir la réussite en contexte scolaire urbain}

À leur arrivée dans le système scolaire, les élèves récemment immigrés font face, de manière variable, à divers défis : deuils et traumatismes (Papazian-Zohrabian, 2016), grands retards scolaires (Armand, 2005a), apprentissage du français (Armand, 2005b), adaptation à un nouveau système scolaire (Rachédi et Vatz Laaroussi, 2016), contrecoups des difficultés d'établissement de la famille (Kanouté, 2007). Cependant, les enjeux suscités par la diversité dans les milieux scolaires montréalais ne sont pas exclusivement liés à la situation d'élèves récemment arrivés. En effet, les défis auxquels certaines familles sont confrontées perdurent parfois d'une génération à l'autre, élargissant ainsi la problématique au-delà de l'immigration récente.

Plusieurs élèves sont donc touchés par un cumul de vulnérabilités socioscolaires étroitement liées à leur parcours migratoire ou à celui de leur famille. Au cours des dernières décennies, différentes initiatives ont été proposées de façon systémique pour soutenir un public scolaire issu de l'immigration au Québec : les classes d'accueil (1969), le Programme d'enseignement des langues d'origine (PELO), la Politique d'intégration et d'éducation interculturelle (1998), etc. (MEQ, 1998 ; Mc Andrew et collab., 2015 ; Mc Andrew et Bakhshaei, 2016). Plus localement, certaines commissions scolaires ou écoles adhèrent également à des pratiques qui visent à soutenir la réussite de ces élèves. Il semble néanmoins que l'adaptation et la prise en compte de la diversité dans les milieux scolaires québécois se fassent à géométrie variable, et qu'il demeure ainsi pertinent de poursuivre la réflexion entamée à ce sujet.

S'intéressant à cette problématique, plusieurs chercheurs québécois (Bakhshaei, 2013; Gosselin-Gagné, 2012 ; Kanouté, Gosselin-Gagné, Guennouni Hassani et Girard, 2016) ont, depuis plusieurs années, questionné des élèves et des familles immigrantes afin de mieux connaître leur vécu socioscolaire et de mettre en exergue les caractéristiques de leur parcours (ex. : défis et tuteurs de résilience). Par exemple, à partir du témoignage d’élèves et de parents récemment immigrés, Gosselin-Gagné (2012) dépeint l'école et les contextes considérés comme étant des tuteurs de résilience potentiels. Jusqu'à maintenant, peu de travaux ont toutefois cherché à dresser un portrait global des caractéristiques des établissements scolaires québécois qui se mobilisent pour s'adapter à la diversité ethnoculturelle en donnant principalement la parole aux membres du personnel scolaire, les principaux concernés. En cohérence avec les éléments de problématique présentés, la question de recherche suivante a ainsi été formulée : quelles sont les caractéristiques des milieux scolaires pluriethniques et défavorisés montréalais qui se mobilisent pour s'adapter à la diversité sociale et ethnoculturelle?

\section{L'éducation inclusive}

Depuis plusieurs décennies, différentes approches théoriques ont été proposées pour « baliser » la gestion de la diversité en milieu scolaire. De plus en plus de chercheurs s'intéressent au paradigme de l'école ou de l'éducation inclusive. Les pratiques d'inclusion désignaient traditionnellement l’intégration dans les classes ordinaires d'élèves 
ayant des handicaps ou des difficultés d'apprentissage (Vienneau, 2002), mais plusieurs auteurs élargissent dorénavant la définition de l'approche inclusive en éducation à la prise en compte et à l'adaptation à la diversité entendue de manière plus large (Ainscow et Miles, 2008 ; Manço, 2016 ; Potvin, 2014 ; UNESCO, 2008 et 2009).

Théoriquement, l'objectif ultime d'une approche inclusive est de promouvoir une éducation qui s'adresse à tous, dans une perspective d'équité et de justice sociale, et dans le but de contrer les inégalités, la discrimination, l'échec et l'exclusion à l'école, mais aussi plus largement dans la société (Ainscow et Miles, 2008 ; Booth et Ainscow, 2002 ; UNESCO, 2008 et 2009). Dans les écrits scientifiques, les milieux scolaires inclusifs sont dépeints comme étant des écoles dans lesquelles interagissent plusieurs acteurs au quotidien qui convergent vers un même objectif: la réussite de l'ensemble des élèves, qu'ils soient en difficulté ou non. La direction d'établissement y est généralement présentée comme le chef d'orchestre de l'organisation : c'est elle qui donne le ton aux pratiques inclusives (Ainscow et Miles, 2008). Les théoriciens de l'approche décrivent le leadership de la direction comme étant engagé, transformatif et partagé (Shields, 2010). Dans de tels milieux scolaires, les différents membres du personnel scolaire partagent des attitudes, des croyances et des valeurs positives vis-à-vis de la diversité. L'un des préceptes fondamentaux d'une éducation inclusive est que c'est à l'école de s'adapter aux besoins des élèves et non l'inverse (Ainscow et Miles, 2008). Les praticiens scolaires se mobilisent donc en ce sens et cherchent à être outillés pour le faire : les pratiques pédagogiques qu'ils privilégient sont flexibles et différenciées et, surtout, adaptées aux besoins (Thomazet, 2006). De plus, les membres des équipes-écoles se respectent et collaborent pour soutenir la réussite éducative (Larivée, Kalubi et Terrisse, 2006). Dans un milieu qui se veut inclusif, les différents membres du personnel cherchent aussi à favoriser un sentiment d'appartenance et à développer l'empowerment (le pouvoir d'agir). II s'agit d'écoles dans lesquelles le climat est généralement positif. Les écrits scientifiques notent également que les milieux scolaires inclusifs valorisent les rapports avec les familles horizontaux et dialogiques (Ainscow et Miles, 2008 ; Booth et Ainscow, 2002) ainsi que le travail continu avec la communauté, qui permet l'accès à une variété de ressources (Loreman, Deppeler et Harvey, 2010).

L'éducation inclusive semble prometteuse pour répondre à différents enjeux en milieu scolaire urbain, et ce, même si, dans les écrits scientifiques recensés, l'approche n'est pas présentée comme une panacée. L'intérêt grandissant pour ce paradigme met en relief la nécessité de repenser un cadre de référence adapté aux défis qui se posent au système scolaire québécois dans sa tentative de trouver une réponse systémique à un public scolaire qui cumule différentes vulnérabilités, parfois liées à leur parcours migratoire ou à celui de leurs parents. En congruence avec cette recension et avec notre question de recherche, cette contribution vise à décrire les caractéristiques d'une école qui se veut inclusive en se mobilisant pour s'adapter à la diversité ethnoculturelle et sociale. Dans cette perspective, quatre objectifs spécifiques ont été établis :

- Objectif 1 : Décrire et analyser le style de leadership de la direction d'une école qui prend en compte la diversité et qui se veut inclusive.

- Objectif 2: Décrire et analyser les pratiques professionnelles spécifiques des enseignants et des autres professionnels d'une école qui prend en compte la diversité et qui se veut inclusive.

- Objectif 3 : Décrire et analyser la dynamique relationnelle générale entre une école qui se veut inclusive et les familles des élèves.

- Objectif 4 : Décrire et analyser la dynamique relationnelle générale entre une école qui se veut inclusive et les ressources de la communauté.

\section{Une ethnographie scolaire}

En vue d'atteindre ces objectifs, j'ai mis en œuvre une recherche qualitative compréhensive adoptant une approche d'inspiration ethnographique (Pepin, 2011; Woods, 1990). L'immersion sur le terrain a semblé indispensable pour répondre aux objectifs de recherche, permettant de produire un tableau détaillé des milieux ciblés incluant les caractéristiques de deux écoles primaires de Montréal sur le plan du leadership de la direction d'établissement, des pratiques et attitudes professionnelles du personnel et des dynamiques relationnelles/partenariales au sein de l'équipe-école. Le fait de choisir deux écoles a permis la récolte de davantage de données. 
Concrètement, pendant une année scolaire, je me suis intégrée au quotidien des écoles participantes, ce qui m’a donné accès à leurs "coulisses », c.-à-d. à ce qui se passe dans des moments informels et qui permet d'éclairer l'objet de recherche. Ces milieux scolaires ont un indice de défavorisation respectif qui oscille entre 9 et 10 d'une année à l'autre, 10 étant le plus haut niveau de défavorisation. De plus, la majorité de leurs élèves sont issus de l'immigration (au-dessus de $85 \%$ de $1^{\text {re }}$ ou de $2^{\text {e }}$ génération). La collecte de données s'est amorcée au mois d'octobre et s'est achevée vers la fin de l'année scolaire. Mon statut d’observateur participant périphérique m'a permis d'assister à une panoplie d'événements et de contextes (cours, assemblées générales, activités parentenfant, dîners dans le salon du personnel, etc.), en plus d'entretenir de nombreuses conversations informelles (Blondin, 2005 ; Lapassade, 2002 ; Pepin, 2011) avec une grande variété d'acteurs : directions, enseignants, parents, acteurs communautaires, etc. Tout au long de l'année, mes notes (observations en dialogues avec des réflexions théoriques) ont été colligées dans un journal ethnographique, afin de conserver des traces écrites de mon passage sur le terrain (Baribeau, 2005). Des entretiens individuels semi-dirigés (Quivy et Van Campenhoudt, 1995) ont également été réalisés auprès d’une variété d'intervenants: quatre directions, dix enseignants, une psychoéducatrice, une technicienne en éducation spécialisée, une responsable du service de garde, une secrétaire et un concierge ; la directrice d'un organisme communautaire partenaire ainsi que trois parents ont également été rencontrés. Un entretien de groupe semi-directif (Quivy et Van Campenhoudt, 1995) a aussi été mené auprès de quatre professionnelles (une psychologue, une orthopédagogue, une orthophoniste et une psychoéducatrice) et d'une technicienne en éducation spécialisée. Les entretiens informels ainsi que l'observation m'ont permis de me familiariser avec les intervenants des écoles participantes et d'obtenir de l'information à divers niveaux. Par exemple, en dînant avec le personnel d'une école à de nombreuses reprises, j'ai été à même de constater la complicité entre les membres de l'équipe. Les entretiens formels, réalisés plus tard dans la collecte de données, m’ont quant à eux permis d'approfondir certaines thématiques et de faciliter ma compréhension de la complexité des milieux participants. Cela m'a permis, entre autres, de croiser le regard des différents acteurs avec le mien afin d'enrichir le portrait brossé de chaque école.

À l'issue de la collecte de données, chaque entretien a été soumis à un processus d'analyse thématique (Paillé et Mucchielli, 2016). Les données d'entretien ont été traitées à la manière d'un codage mixte (Van der Maren, 1996). Le travail a été amorcé par le codage des entretiens réalisés avec les directions d'écoles. Ensuite, je me suis basée sur cette liste de codes provisoire (ex.: Distribuer le leadership; Collaborer et travailler en équipes interdisciplinaires) pour coder les autres entretiens (enseignants, professionnels, etc.), tout en demeurant ouverte à la création de nouveaux codes ou à la modification de certains d'entre eux (ex.: Différencier les pratiques pédagogiques; Faire preuve de flexibilité). Les catégories créées à l'aide du logiciel QDA Miner ont donc été remaniées au fur et à mesure du traitement des données. Le codage des entretiens a été en partie réalisé à partir de thèmes liés à des dimensions inspirées de ma problématique et de mon cadre théorique (ex. : Cumul d'emplois précaires ; Isolement social des familles). Tout au long de la phase de codification des données, j'ai également porté attention aux catégories émergentes et significatives révélées par le corpus et non anticipées par la recension (Lecompte et Schensul, 2010). Par exemple, plusieurs acteurs ont affirmé que les nombreuses transitions que vivent les élèves constituaient un défi spécifique au milieu, alors que cet aspect n’avait pas été révélé par la recension des écrits.

L'analyse de mon journal ethnographique, quant à elle, a été amorcée parallèlement à la collecte de données, à l'aide d'itérations entre les écrits scientifiques et le terrain (Baribeau, 2009 ; Laperrière, 1997). À plusieurs reprises, j'y ai consigné des notes théoriques (Martineau, 2005), ce qui m’a permis d'établir des liens entre ce que j'observais et entendais dans les milieux participants et la théorie recensée (ex. : J'ai l'impression que les directions de chaque école ont des styles de leadership différents selon les contextes et les situations [Bass, 1990] [extrait du journal, 4 février 2016]). Les notes de terrain ainsi que les autres documents amassés (projets éducatifs, courriels) m'ont permis d'enrichir le portrait des écoles (voir Gosselin-Gagné, 2018, pour davantage d'information). À la suite de l'analyse de l'ensemble des données, plusieurs caractéristiques spécifiques des défis rencontrés par ces milieux et des leviers d'intervention que les acteurs mettent en œuvre au quotidien ont été mises en lumière.

Alterstice - Revue Internationale de la Recherche Interculturelle, vol. $8, n^{\circ} 2$ 


\section{L'intervention en contexte de défavorisation et de pluriethnicité : des défis aux leviers}

Dans les sections qui suivent est d'abord brossé le portrait des différents défis propres aux milieux scolaires participants qui ont été observés ou rapportés, à savoir la précarité, les difficultés psychosociales, les traumatismes et le décodage d'une nouvelle culture scolaire. Ensuite, en cohérence avec les quatre objectifs de recherche présentés plus haut, sont décrits les leviers d'intervention que l'analyse des données a mis en exergue.

\section{Les défis}

\section{La précarité et les difficultés psychosociales}

Au Québec comme ailleurs, les effets de la pauvreté sur l'expérience socioscolaire ont fait l'objet de différentes publications. Mittler (2000), par exemple, a documenté les impacts de la précarité sur le développement des enfants ainsi que sur leur parcours scolaire. D'après l'auteur, ceux-ci seraient plus nombreux à vivre dans des appartements bruyants et exigus, ce qui peut se traduire par une difficulté à réussir ; ces affirmations rejoignent les propos de plusieurs personnes avec qui je me suis entretenue sur le terrain. Certains ont mentionné que l'environnement familial dans lequel vivent certains élèves nuit à leur concentration et à leur disposition à apprendre, parce que leur hygiène de sommeil est affectée, par exemple. D'autres défis sur le plan de l'hygiène de vie ont également été soulignées. Par exemple, des acteurs avec qui je me suis entretenue estiment que certains enfants ne mangent pas à leur faim et que leurs lunchs sont peu soutenants ou nutritifs.

Des personnes rencontrées ont également souligné le fait qu'il y a des élèves qui évoluent dans des familles aux prises avec des difficultés psychosociales qui peuvent se traduire par de la négligence notamment : "Je l'observe surtout par les vêtements que les enfants portent. On peut voir un peu de négligence : des vêtements sales, trop petits ou des souliers trop grands" (une enseignante). Selon Mittler (2000), les contraintes financières peuvent induire du stress chez les adultes qui, en retour, ont tendance à éprouver des difficultés sur le plan de l'exercice de la parentalité. Dans une école, la directrice adjointe a tenu les propos suivants : "C'est très difficile d'intervenir parce qu'on parle d'une relation parent-enfant qui est déficiente, d'un abandon affectif. Des fois, même si le parent est présent physiquement, l'enfant vit l'abandon ». Les observations du personnel scolaire rencontré se sont souvent rapprochées des propos d'auteurs qui soulignent les différents impacts potentiels de la négligence: retards psychomoteurs, cognitifs ou socioaffectifs, troubles du comportement, échec scolaire et autres (Hildyard et Wolfe, 2002). Dans les écoles participantes, plusieurs ont rapporté les difficultés ou les retards observés chez certains élèves du milieu au niveau de la socialisation, de la motricité globale ou fine, de l'attention-concentration, de l'organisation, des difficultés langagières, etc. : «Quand je suis arrivée ici, la première chose qui m'a frappée, c'est que certains élèves étaient très peu stimulés. J'étais en première année et on avait un élève qui n'avait pas fait de maternelle et qui ne savait pas comment tenir un crayon pour colorier » (une enseignante). Selon Kamanzi, Zhang, Deblois et Deniger (2007), les conditions socioéconomiques précaires ont tendance à limiter l'accès aux ressources matérielles et culturelles (ex. : livres, lecture, jeux, théâtre, etc.) qui soutiennent en partie l'expérience ainsi que la réussite scolaire. Des intervenants que j'ai côtoyés estiment effectivement que les pratiques de lecture sont peu répandues dans certaines familles et que l'accès aux livres y est restreint. D'autres pensent que certains élèves souffrent de défavorisation socioculturelle en partie parce qu'ils sortent peu de la maison. Quoi qu'il en soit, la précarité et les difficultés psychosociales auxquelles certaines familles du milieu sont aux prises induisent différents facteurs de risque pour ces enfants.

\section{Les traumatismes et le décodage d'une nouvelle culture scolaire}

Certains élèves souffrent de traumatismes parce qu'ils ont été témoins ou victimes de violence. Leurs blessures peuvent être physiques (ex. : handicaps et invalidités) ou psychiques (ex. : perte d'un membre de leur famille) (Papazian-Zohrabian, 2016). Tout au long de l'année, diverses personnes avec qui je me suis entretenue ont évoqué les difficultés liées aux conditions prémigratoires vécues par ces familles. Certains ont raconté le cas d'élèves qui ont été témoins ou victimes de situations de violence, évoquant parfois des histoires traumatiques: « J'en ai un dans ma classe en ce moment, il a vu des gens se faire tuer devant lui. [...] J'ai aussi eu des enfants qui, avant d'arriver ici, ont toujours vécu dans des camps de réfugiés » (un enseignant). À travers différentes conversations formelles et informelles, on m'a également raconté l'histoire d'élèves qui, avant d'arriver au Canada, ont été euxmêmes blessés lors de différents événements : " Il y a une jeune dont le père et le frère ont été assassinés dans la 
voiture. Elle aussi, ils lui ont tiré dessus. Elle a perdu un poumon et on lui a aussi enlevé une partie du muscle au niveau du bras" (un directeur). Papazian-Zohrabian (2013) explique que, chez les enfants, les deuils et les traumatismes peuvent avoir un grand impact sur la capacité d'adaptation ainsi que l'expérience scolaire. Dans une publication plus récente (2016), l'auteure soutient que certains d'entre eux éprouvent des difficultés de comportement et d'apprentissage, un manque de motivation et de disponibilité cognitive et conaissent une diminution de leur rendement scolaire, qui conduit parfois à l'échec.

Dans une école, plusieurs se sont d'ailleurs avoués préoccupés par les besoins de plus en plus fréquents de services en psychologie chez les enfants du milieu. Aussi, le nombre d'élèves diagnostiqués comme handicapés ou en difficulté d'adaptation ou d'apprentissage (HDAA) étant élevé, les défis le sont également. D’après un directeur, certains enfants cumulent plusieurs vulnérabilités, qui expliquent en partie leurs difficultés psychosociales : "Ce sont des jeunes qui sont parfois très sanguins, je dirais. Ils ont connu des choses, ils ont connu la guerre, donc, ils sont très réactifs ». D'autres acteurs scolaires ont souligné les troubles de comportement qu'on retrouve chez plusieurs élèves: "Les enfants se fâchent rapidement et ils ont de la misère à se calmer. Tu sais, ce n'est pas quelque chose que je voyais dans mon ancien milieu, mais que je vois ici » (une directrice adjointe).

Plusieurs personnes rencontrées ont également souligné les difficultés d'adaptation chez des élèves ayant été scolarisés dans des systèmes scolaires très différents du modèle québécois. La directrice adjointe d'une école a expliqué que des enfants, bien qu'ils soient francophones, ont des acquis et des expériences qui les situent à une certaine distance du système scolaire québécois, ce qui pose différents défis. À son avis, les difficultés d'adaptation peuvent également se traduire sur le plan social. D’après Rachédi et Vatz Laaroussi (2016), certains enjeux d'adaptation sont rattachés aux nouvelles méthodes pédagogiques ou à des différences culturelles lorsque les valeurs, les normes et les coutumes de l'école québécoise se situent à distance de ce qu'ils ont connu jusque-là. La directrice adjointe a également souligné, au passage, les chocs culturels liés au système d'éducation que vivent certains parents immigrants récents ainsi que les défis que cette situation pose pour l'école.

Le français n'est pas la langue maternelle de plusieurs élèves des écoles participantes et il ne s'agit pas non plus de la langue d'usage à la maison. Sans prétendre qu'il s'agit d'un " problème ", l'orthophoniste d'une école a expliqué qu'il peut être plus difficile d'évaluer si les difficultés d'un enfant sont dues à la maîtrise de la langue ou à autre chose. La psychologue d'une école a, elle aussi, souligné le défi de poser un diagnostic lorsqu'un élève ou ses parents ne maîtrisent pas le français. Ces propos rejoignent ceux d'auteurs qui ont observé la difficulté à évaluer les besoins liés à l'appropriation d'une langue et ceux suscités par des difficultés d'apprentissage (Borri-Anadon, Savoie-Zajc et Lebrun, 2015). Par ailleurs, quelques intervenants ont soutenu le fait que certains enfants ont peu d'occasions de pratiquer cette langue en dehors de l'école, ce qui fait qu'ils éprouvent parfois de la difficulté et que le processus d'acquisition de la langue est plus long. Or, comme le soulignent plusieurs auteurs (Armand, 2005b ; Bouchamma, 2009), l'apprentissage ainsi que la maîtrise subséquente de la langue d'enseignement sont essentiels à l'intégration socioscolaire.

\section{Les leviers pour intervenir}

Sensibles au cumul de vulnérabilités que vivent certains élèves, les intervenants des écoles ciblées par ma recherche tentent de s'adapter à leurs besoins et de se mobiliser afin de soutenir leur réussite éducative, efforts que j'ai documentés en relation avec quatre dimensions : le leadership des directions d'école, le climat socioprofessionnel et les pratiques qui visent les élèves, les relations école-familles immigrantes et la collaboration des écoles avec les ressources communautaires qui les entourent.

\section{Le leadership des directions d'école}

L'analyse menée sur les données a permis d'identifier différentes caractéristiques des directions des écoles participantes sous l'angle du paradigme de l'éducation inclusive (Bélanger, 2010 ; Riehl, 2000 ; Shields, 2010). Les directions et les directions adjointes que j'ai côtoyées sont engagées et bienveillantes, comme des parents qui prennent soin de leurs enfants : "Ici, la direction, c'est comme une petite famille... J'ai pas vu ça ailleurs. [...] Moi, depuis que je suis ici, je vois que la direction essaie d'amener les choses pour le bien-être des élèves. II y a beaucoup de changements " (un concierge). Ce sont des leaders qui se mobilisent au quotidien pour trouver des 
moyens de répondre aux besoins sur différents plans. Leur principale préoccupation est de mettre en place tout ce qu'ils peuvent pour soutenir la réussite éducative et orienter leur équipe respective vers cet objectif-là. Les directions ont souvent insisté sur le fait qu'un enfant qui a de la difficulté doit être pris en charge, peu importe ses besoins, et que les différents membres du personnel doivent se sentir concernés. Lors d'une rencontre à laquelle j'ai assisté, la première question posée par le directeur concernant une enfant en difficulté a été : "Qu'est-ce qu'on pourrait faire pour la motiver? " (extrait du journal, 8 février 2016). Les directions des écoles participantes m'ont expliqué qu'elles ont souhaité travailler dans un milieu comme le leur pour faire une différence auprès d'élèves plus vulnérables. Elles sont sur tous les fronts et rarement assises dans leur bureau. On dit qu' « elles marchent leur école ». De manière générale, ces directions sont curieuses d'en savoir plus sur les enjeux liés à la pauvreté et à l'immigration; elles s'intéressent aux recherches à ce sujet et en informent leur équipe de manière formelle et informelle. Dans une école, un directeur m'a confié qu'il sentait qu'il avait encore beaucoup de choses à apprendre concernant les relations interculturelles, mais qu'il était très motivé à y arriver.

Les directions que j'ai côtoyées ont une approche critique et, dans certaines situations, elles tentent de faire preuve de leadership transformatif, un style de leadership fortement rattaché à la théorie de l'éducation inclusive (Shields, 2010). Ces personnes souhaitent être des agents de changement dans leur milieu et adoptent des pratiques et des discours cohérents à ce sujet. Par ailleurs, les directions tentent elles aussi de se remettre en question pour améliorer leurs propres pratiques (ex. : un directeur demande chaque année à son équipe d'évaluer son travail). Les directions des milieux participants tentent également de distribuer le leadership et de favoriser des relations professionnelles horizontales, des caractéristiques qu'Ainscow et Miles (2008) associent aux directions en milieux scolaires inclusifs. Dans une école, l'équipe de direction s'est affairée à déconstruire la hiérarchie qui s'était installée entre certains membres du personnel en organisant une activité de team building et en sensibilisant l'équipe à l'importance de travailler ensemble.

De manière générale, l'équipe de direction des deux écoles m’a semblé appréciée et respectée, autant des jeunes que des adultes. En les côtoyant, j'ai eu l'impression qu'elles exercent leur leadership au quotidien d'une manière humaine, ce qui se reflète sur le climat d'école.

\section{Le climat socioprofessionnel et les pratiques qui visent les élèves}

L'analyse menée a permis de faire émerger différentes caractéristiques des écoles participantes quant aux dynamiques socioprofessionnelles, aux attitudes et aux pratiques qui visent les élèves, toujours en les analysant sous l'angle du paradigme de l'éducation inclusive (Ainscow et Miles, 2008 ; Booth et Ainscow, 2002 ; Loreman, Deppeler et Harvey, 2015 ; Potvin, 2014 ; Riehl, 2000). Sur le plan des relations professionnelles, j'ai recensé différents aspects qui semblent avoir un impact sur le climat des écoles. Certaines personnes ont affirmé que le travail avec des enfants dont le vécu est difficile demande de se soutenir et de prendre soin les uns des autres: «En milieu défavorisé, le côté humain, c'est très important. Et c'est pas juste avec tes élèves; c'est avec le personnel aussi. [...] Tu sais, il faut faire attention à nous parce que si l'équipe est plus là, on n'arrivera pas à aider les enfants " (une directrice adjointe). Les gens que j'ai côtoyés tentent, de manière générale, de se respecter, de communiquer et de travailler en équipe, des caractéristiques qui font écho à la théorie de l'éducation inclusive (Larivée, Kalubi et Terrisse, 2006; Manço, 2016). Une enseignante a tenu les propos suivants: "Ce sont des enseignantes qui collaborent, qui sont engagées [...] c'est la première fois que j'arrive dans une école où les enseignantes travaillent en équipe, main dans la main, tout le monde sur un pied d'égalité, puis que tout le monde a à cœur le succès des élèves ". Dans une école, les membres du personnel dînent souvent ensemble, ce qui permet des échanges positifs et des rires, dans une ambiance informelle. Cette complicité semble soutenir les interventions menées au quotidien et avoir un impact sur le climat scolaire. Plusieurs personnes avec qui je me suis entretenue ont aussi souligné l'importance de travailler de façon interdisciplinaire, renvoyant ainsi à un principe de l'éducation inclusive (Ainscow et Miles, 2008). Par ailleurs, certains ont mis l'accent sur l'accueil des nouveaux enseignants, un autre moyen identifié par Booth et Ainscow (2002) pour soutenir la qualité du climat.

Pendant l'année, j'ai donc côtoyé des personnes qui font preuve de différentes qualités " humaines ». Elles sont empathiques, ont des attitudes positives et des connaissances concernant la diversité ; elles sont curieuses du vécu des élèves et de la diversité en général. Selon Ainscow et Miles (2008), il s'agit de caractéristiques présentes chez 
les intervenants qui souhaitent être inclusifs à la diversité. Plusieurs ont parlé de l'amour des voyages ou de la volonté d'apprendre des mots dans différentes langues. Certains ont affirmé qu'ils tentent de se renseigner à propos des enjeux du milieu afin d'être outillés pour intervenir face à différentes situations. Plusieurs personnes m'ont expliqué qu'elles essaient d'être réflexives et de se décentrer sur le plan socioculturel. D'autres posent agissent pour soutenir la motivation et le sentiment d'appartenance. Par exemple, certains ont parlé de l'importance d'insuffler de l'espoir aux élèves (ex.: en leur présentant des modèles de réussite) pour qu'ils ne pensent pas leur situation (ex. : la précarité liée aux difficultés d'établissement de leur famille) comme étant une fatalité. De manière générale, les gens que j'ai rencontrés sont engagés et dévoués : "Je ne serais jamais capable de dire : "J'appellerai pas parce que ce n'est pas sur mes heures." [...] On ne peut pas "puncher" ici. Ça ne marche pas comme ça. II y a des responsabilités qui vont au-delà des heures de classe " (une enseignante). Ils savent s'adapter rapidement et sont flexibles : «Il faut toujours être vite, rapide, et offrir des solutions de rechange... Être capable de s'ajuster [...] puis ne pas être fermé parce qu'y arrive tellement d'imprévus que, même si t'as un horaire, il faut que tu te revires de bord rapidement quand y'a quelque chose " (une psychoéducatrice). Plusieurs personnes m'ont expliqué qu'elles tentent d'être créatives et qu'elles doivent parfois s'aventurer "hors des sentiers battus » pour répondre à certains besoins. Selon Ainscow et Miles (2008), les milieux scolaires inclusifs font souvent preuve de créativités afin de soutenir le plus grand nombre d'élèves.

Plus concrètement, en ce qui concerne les pratiques qui visent à soutenir les élèves, chaque école essaie de combler les besoins de base : nourriture, vêtements, fournitures scolaires. Lorsque des enfants arrivent, au début ou en cours d'année, on tente de les accueillir explicitement et on cherche à en savoir davantage sur leur vécu, leurs défis et leurs forces aussi. Que ce soit en lien avec un parcours migratoire ou non, certains enfants du milieu vivent ou ont vécu des situations difficiles :

Si je retrouve un enfant qui a vécu la guerre, souvent caché parce qu'il entend des bruits, ce n'est pas nécessairement qu'il a un trouble de comportement. II ne faut pas sonner l'alarme tout de suite. Il faut vraiment aller voir le passé de l'enfant. J'en avais un en sixième année qui venait juste d'arriver. II avait vu ses parents morts. Cet enfant-là, il frappait tout le monde. De lui donner des « fiches bleues » sans essayer de comprendre d'où il vient, ça ne règle rien. Donc, c'est important d'aller voir le passé, de poser des questions, puis d'essayer d'aller connaître tous les facteurs de risque. (une enseignante)

De manière générale, les membres du personnel sont donc très sensibles au vécu des élèves et tentent de mettre en place un climat de sécurité physique et psychologique et de soutenir l'implication et le leadership des élèves, autant de caractéristiques qui, selon Bélanger (2010), sont présentes dans les milieux scolaires inclusifs.

\section{Les relations école-familles immigrantes}

J'ai également pu relever différentes pratiques déployées dans les écoles participantes pour soutenir la relation avec les familles; elles ont été analysées et comparées au modèle théorique de l'éducation inclusive (Ainscow et Miles, 2008; Booth et Ainscow, 2002 ; Riehl, 2000). Dans les écoles participantes, plusieurs acteurs ont abordé l'importance d'employer une variété de moyens pour communiquer avec les parents qui ne maîtrisent pas le français : faire appel à des interprètes, utiliser différentes langues ou mettre à profit la diversité ethnoculturelle et linguistique des collègues. Selon Booth et Ainscow (2002), il s'agit de préoccupations que doivent avoir des intervenants qui souhaitent être inclusifs à la diversité. La plupart des acteurs côtoyés tentent également d'entrer régulièrement en contact avec les parents et font preuve de souplesse. Lorsque nécessaire, ils appellent le soir ou la fin de semaine pour rejoindre certains parents, car ils se disent sensibles à la réalité de ceux dont les horaires de travail sont atypiques. Plusieurs personnes avec qui je me suis entretenue ont mis l'accent sur l'importance d'accueillir les familles quand elles viennent à l'école : "Je trouve ça très satisfaisant de les accueillir, de prendre contact avec eux... Ça fait tellement une différence ! Ils sont stressés ces parents-là, surtout parce qu'ils ne savent peut-être pas comment [l'école] fonctionne " (une directrice adjointe). Les directions des deux milieux profitent de chaque moment où elles croisent les parents afin de créer des liens et de démystifier leur rôle. Dans une école, lorsque des familles (immigrantes pour la plupart) arrivent en cours d'année, différents intervenants les accueillent en leur faisant visiter l'école, en les présentant aux membres du personnel scolaire et en les mettant en contact avec les ressources du quartier, par exemple. Selon Booth et Ainscow (2002), on retrouve ce type de pratiques dans les écoles qui souhaitent être inclusives à la diversité. Les parents des deux milieux sont souvent invités en dehors des rencontres formelles et leur implication est encouragée de différentes manières, comme le préconisent 
notamment Larivée, Kalubi et Terrisse (2006). Dans une école, des enseignantes ouvrent les portes de leur classe à des parents bénévoles, une pratique qui semble avoir des répercussions intéressantes sur différents plans : «J'ai vu comment mieux aider mon enfant à la maison, surtout pour les devoirs. [...] J'ai beaucoup aimé ! Ça m'a ouvert les yeux et j'ai appris beaucoup de choses. De voir comment l'enseignant enseigne, ça permet de faire un lien avec ce qu'on fait après à la maison » (un parent). Dans cette école, on offre également des cours de francisation. La directrice estime qu'il est important d'ouvrir les portes de l'école afin que les familles s'y sentent à leur place.

De manière générale, la plupart des personnes prennent le temps d'écouter les parents. Ces derniers sont parfois inquiets pour leurs enfants qui ont vécu des choses difficiles et ont besoin d'être rassurés sur différents plans. Plusieurs intervenants des écoles participantes tentent d'outiller les parents de façon horizontale, c.-à-d. qu'ils offrent des conseils ou les réfèrent aux organismes communautaires, mais n'essaient pas de leur imposer une façon d'élever leur enfant. Enfin, certaines personnes que j'ai côtoyées choisissent d'accompagner des familles au-delà du mandant initial de l'école parce qu'elles pensent que cela fait une différence.

La collaboration des écoles avec les ressources communautaires qui les entourent

J'ai pu également mettre en exergue différentes pratiques qui visent à créer et à soutenir la collaboration avec divers organismes communautaires; ces pratiques ont été analysées à travers les lunettes des préceptes de l'éducation inclusive (Ainscow et Miles, 2008 ; Loreman, Deppeler et Harvey, 2010). Les écoles qui ont participé à ma recherche sont conscientes du fait que, pour soutenir les élèves et les familles, il est essentiel qu'elles soient en contact avec les ressources communautaires, des observations qui font écho à la théorie de l'éducation inclusive (Loreman, Deppeler et Harvey, 2010). Un directeur a tenu les propos suivants : «II faut les consulter parce qu'ils ont souvent des pistes de solution auxquelles on n'aurait pas pensé tout ça pour aboutir à la réussite du plus grand nombre de jeunes; la réussite scolaire, mais aussi éducative, sociale... » À ce sujet, la direction d'une école et une psychoéducatrice m'ont souvent parlé souvent de l'importance de faire connaître les ressources communautaires à leurs collègues pour une plus vaste prise en charge des besoins des élèves.

Différents intervenants ont affirmé qu'ils vont rendre visite à divers organismes afin de se renseigner sur les services offerts et de mieux se connaître pour travailler ensemble. Les milieux participants invitent aussi les partenaires de la communauté à l'école quand il y a des séances d'information avec les parents: "Les parents peuvent poser des questions et savent que les organismes existent. Et ils parlent à quelqu'un, à une personne ; c'est plus rassurant qu'une carte ou juste un dépliant » (une psychoéducatrice). Mon passage sur le terrain m’a également permis de participer à une autre initiative de rapprochement: une rencontre conviviale réunissant différents acteurs de la communauté (organismes, policier sociocommunautaire, etc.) a été organisée afin de travailler en plus étroite collaboration pour soutenir les familles du milieu. Enfin, dans une des écoles participantes, quelques intervenants ont fait parvenir des cartes de Noël à différents partenaires afin de les remercier de leur collaboration dans le but de se rapprocher et de créer des liens.

\section{La recherche au service de la mise en œuvre d'une école inclusive}

L'objectif de ma recherche doctorale consistait à brosser un portrait de milieux scolaires montréalais qui se mobilisent pour s'adapter aux besoins en contexte de défavorisation et de pluriethnicité. Pour ce faire, j'ai recensé différentes caractéristiques de deux écoles primaires de Montréal quant au leadership de leur direction, aux dynamiques socioprofessionnelles entre les membres du personnel et aux pratiques qui visent à soutenir le bienêtre ainsi que la réussite des élèves, aux relations avec les parents et à la collaboration avec les ressources communautaires. L'ensemble de ces caractéristiques a été observé et analysé à travers les lunettes théoriques de l'éducation inclusive. Cette recherche a permis de mettre en lumière comment une éducation inclusive peut être mise en œuvre en contexte scolaire québécois afin d'intervenir auprès d'un public scolaire parfois vulnérable et, ainsi, de soutenir la réussite du plus grand nombre d’élèves.

L'objectif de brosser un portrait global des écoles participantes semble à la fois une force et une limite, puisqu'il a fait en sorte que je me suis moins concentrée sur certaines pratiques. Notamment, j'aurais souhaité documenter davantage le travail des enseignants en classe, car il s'agit d'un élément essentiel pour enrichir la formation initiale des maîtres ainsi que leur formation continue ; j'estime ainsi qu'il serait intéressant de poursuivre un tel objectif 
dans une recherche ultérieure. Une autre direction qui semble prometteuse serait de conduire une recherche avec des paramètres semblables à celle-ci, mais dans des écoles secondaires, car certains enjeux diffèrent, notamment en relation avec l'âge d'entrée dans le système scolaire et la capacité d'adaptation (Bouchamma, 2009), l'implication des parents dans le parcours scolaire (Marcotte, Fortin, Cloutier, Royer et Marcotte, 2005) et les interventions communautaires (Bilodeau, Lefebvre, Deshaies, Gagnon, Bastien, Bélanger, Potvin et Carignan, 2011). Enfin, il faudrait aussi chercher à savoir quels sont les impacts concrets de ces pratiques dites inclusives sur les élèves. Quoi qu'il en soit, dans le contexte actuel où des centaines de familles s'établissement chaque année au Québec, il est essentiel de poursuivre les recherches afin de mieux comprendre comment mettre en œuvre une éducation inclusive au sein des écoles, dans le but de soutenir la réussite de tous.

\section{Références bibliographiques}

Ainscow, M., et Miles, S. (2008). Vers une éducation pour l'inclusion pour tous : prochaine étape ? Perspectives, XXXVIII(1), 20-47. https://www.gcedclearinghouse.org/sites/default/files/resources/170059fre.pdf\#page=20

Armand, F. (2005 a). Capacités métalinguistiques d'élèves immigrants nouvellement arrivés en situation de grand retard scolaire. Revue des sciences de l'éducation, 31(2), 441-469.

Armand, F. (2005 b). Les élèves immigrants nouvellement arrivés et l'école québécoise. Santé, société et solidarité, 1, 141-152. https://www.persee.fr/doc/oss_1634-8176_2005_num_4_1_1035

Bakhshaei, M. (2013). L'expérience socioscolaire d'élèves montréalais originaires de l'Asie du Sud: dynamiques familiales, communautaires et systémiques (Thèse de doctorat, Université de Montréal, Montréal). https://papyrus.bib.umontreal.ca/xmlui/bitstream/handle/1866/10117/Bakhshaei_Mahsa_2013_these.pdf

Baribeau, C. (2005). Le journal de bord du chercheur. Recherches qualitatives, hors-série(2), 198-114.

Baribeau, C. (2009). Analyse des données des entretiens de groupe. Recherches qualitatives, 28(1), $133-148$.

Bélanger, S. (2010). Attitudes des différents acteurs scolaires à l'égard de l'inclusion. Dans N. Rousseau (dir.), La pédagogie de l'inclusion scolaire : pistes d'action pour apprendre tous ensemble (2 éd., p. 111-132). Québec : Presses de l'Université du Québec.

Bilodeau, A., Lefebvre, C., Deshaies, S., Gagnon, F., Bastien, R., Bélanger, J., Potvin, M., et Carignan, N. (2011). Les interventions issues de la collaboration école-communauté dans quatre territoires montréalais pluriethniques et défavorisés. Service social, 57(2), 37-54.

Blondin, D. (2005). L'observation en situation en milieu primaire : dépasser les contraintes, enrichir la recherche. Recherches qualitatives, hors-série (2), 18-37.

Booth, T., et Ainscow, M. (2002). Index for inclusion : Developing learning and participation in schools. Bristol (Royaume-Uni) : Centre for Studies on Inclusive Education (CSIE). https://www.jugendfuereuropa.de/downloads/4-20-3946/Index\%20English.pdf

Borri-Anadon, C., Savoie-Zajc, L., et Lebrun, M. (2015). Pratiques évaluatives des orthophonistes scolaires à l'égard des élèves de minorités culturelles : différenciation, uniformisation et normalisation. Recherches et éducations, 14, 81-92.

Bouchamma, Y. (2009). La réussite scolaire des élèves immigrants : facteurs à considérer. Vie pédagogique, 152.

Gosselin-Gagné, J. (2012). Résilience scolaire chez les élèves allophones du primaire récemment immigrés (Mémoire de maîtrise, Université de Montréal, Montréal). https://papyrus.bib.umontreal.ca/xmlui/bitstream/handle/1866/8517/GosselinGagne_Justine_2012_memoire.pdf

Gosselin-Gagné, J. (2018). L'éducation inclusive comme perspective pour comprendre la mobilisation d'écoles primaires montréalaises qui conjuguent défavorisation et défis relatifs à la diversité ethnoculturelle (Thèse de doctorat, Université de Montréal, Montréal).

https://papyrus.bib.umontreal.ca/xmlui/handle/1866/21215

Alterstice - Revue Internationale de la Recherche Interculturelle, vol. $8, n^{\circ} 2$ 
Hildyard, K. L., et Wolfe, D. A. (2002). Child neglect: Developmental issues and outcomes. Child Abuse and Neglect, 26(6-7), 679-695.

Kamanzi, C., Zhang, X. Y., Deblois, L., et Deniger, M.-A. (2007). L'influence du capital social sur la formation du capital humain chez les élèves résilients de milieux socioéconomiques défavorisés. Revue des sciences de l'éducation, 33(1), 127-145.

Kanouté, F. (2007). Intégration sociale et scolaire des familles immigrantes au Québec. Une prise en compte globale des familles. Informations sociales, 7(143), 64-74.

Kanouté, F., Gosselin-Gagné, J. Guennouni Hassani, R., et Girard, C. (2016). Points de vue d’élèves issus de l'immigration sur leur expérience socioscolaire en contexte montréalais défavorisé. Alterstice, 6(1), 13-25.

Lapassade, G. (2002). Observation participante. Vocabulaire de psychosociologie, ERES, hors collection, $375-390$. http://vadeker.net/corpus/lapassade/ethngr1.htm

Laperrière, A. (1997). La théorisation ancrée (grounded theory) : démarche analytique et comparaison avec d'autres approches apparentées. Dans J. Poupart, J.-P. Deslauriers, L.-H. Groulx, A. Laperrière, R. Mayer et A. P. Pires (dir.), La recherche qualitative : enjeux épistémologiques et méthodologiques (p. 309-340). Montréal : Gaëtan Morin Éditeur.

Larivée, S. J., Kalubi, J.-C. et Terrisse, B. (2006). La collaboration école-famille en contexte d'inclusion : entre obstacles, risques et facteurs de réussite. Revue des sciences de l'éducation, 32(3), 525-543.

LeCompte, M. D., et Schensul, J. J. (2010). Designing and conducting ethnographic research: An introduction. Toronto : AltaMira Press.

Loreman, T., Deppeler, J., et Harvey, D. (2010). Inclusive education: Supporting diversity in the classroom (2 éd.). New York : Routledge.

Manço, A. (2016). Vivre et travailler ensemble à l'école de l'hyperdiversité. Diversités et citoyennetés. La lettre de I'IRFAM, 44-45, 9-14.

Marcotte, J., Fortin, L., Cloutier, R., Royer, É., et Marcotte, D. (2005). Évolution de l'engagement parental auprès des élèves en difficulté de comportement et des élèves ordinaires au début du secondaire. Nouveaux cahiers de la recherche en éducation, 8(2), 47-56.

Martineau, S. (2005). L'observation en situation : enjeux, possibilités et limites. Recherches qualitatives, horssérie(2), 5-17.

Ministère de l'Éducation du Québec (MEQ). (1998). Une école d'avenir. Politique d'intégration scolaire et d'éducation interculturelle. Québec : MEQ.

Mc Andrew, M. et l'équipe du GRIÉS (dir.) (2015). La réussite éducative des élèves issus de l'immigration : dix ans de recherche et d'intervention au Québec. Montréal : Presses de l'Université de Montréal.

Mc Andrew, M., et Bakhshaei, M. (2016). La scolarisation des élèves issus de l'immigration et l'éducation interculturelle : historique, situation actuelle et principaux défis. Dans M. Potvin, M.-O. Magnan et J. Larochelle-Audet (dir.), La diversité ethnoculturelle, religieuse et linguistique en éducation : théorie et pratique (p. 19-40). Anjou : Fides.

Mittler, P. (2000). Working towards inclusive education. London : David Fulton Publishers.

Paillé, P. et Mucchielli, A. (2016). L'analyse qualitative en sciences humaines et sociales (4 éd.). Paris : Armand Colin.

Papazian-Zohrabian, G. (2013). Le deuil traumatique chez l'enfant et son influence sur la construction de son identité. Revue québécoise de Psychologie. 34(2), 83-100.

Papazian-Zohrabian, G. (2016). Les jeunes réfugiés et les enfants de la guerre à l'école québécoise. Dans M. Potvin, M.-O. Magnan et J. Larochelle-Audet (dir.), La diversité ethnoculturelle, religieuse et linguistique en éducation : théorie et pratique (p. 183-196). Anjou : Fides. 
Pepin, M. (2011). L'ethnographie scolaire : comprendre quoi, comment et pour qui ? Recherches qualitatives, 10, 30-46.

Potvin, M. (2014). Diversité ethnique et éducation inclusive : fondements et perspectives. Éducation et sociétés, 1(33), 185-202.

Quivy, R., et Campenhoudt, V. (1995). Manuel de recherche en sciences sociales (2 éd.). Paris : Dunod.

Rachédi, L. et Vatz Laaroussi, M. (2016). Les processus migratoires : revisiter les concepts de base à la lumière des réalités familiales et migratoires contemporaines. Dans M. Potvin, M.-O. Magnan et J. Larochelle-Audet (dir.), La diversité ethnoculturelle, religieuse et linguistique en éducation : théorie et pratique (p. 70-78). Anjou, Québec : Fides.

Riehl, C. J. (2000). The principal's role in creating inclusive schools for diverse students: A review of normative, empirical, and critical literature on the practice of educational administration. Review of Educational Research, 70(1), 55-81.

Shields, C. M. (2010). Transformative leadership: Working for equity in diverse contexts. Educational Administration Quarterly, 46(4), 558-589.

Thomazet, S. (2006). De l'intégration à l'inclusion. Une nouvelle étape dans l'ouverture de l'école aux différences. Le français aujourd'hui, 1(152), 19-27.

UNESCO. (2008). L'Éducation pour l'inclusion : la voie de l'avenir. Conférence internationale de l'éducation, $48^{e}$ session. http://www.ibe.unesco.org

UNESCO. (2009). Vers une école inclusive et une amélioration de l'apprentissage : synthèse des résultats d'études de cas menées dans différents pays. http://unesdoc.unesco.org/images/

Van der Maren, J.-M. (1996). Méthodes de recherche pour l'éducation (2 éd.). Montréal/Bruxelles : Presses de l'Université de Montréal et De Boeck.

Vienneau, R. (2002). Pédagogie de l'inclusion : fondements, définition, défis et perspectives. Éducation et francophonie, $X X X(2)$. https://www.acelf.ca/c/revue/revuehtml/30-2/10-vienneau.html

Woods, P. (1990). L'ethnographie de l'école. Paris : Armand Colin. 